Research article

\title{
A comparative study among using of Ketamine, Tramadol, and Paracetamol in three injectable anesthetic protocols for induction of general anesthesia in sheep
}

\author{
Ali Ismail Jassim \\ Thaier Alwan Abid \\ Department of Surgery, College of Veterinary Medicine, University of Al-Qadisiyah, Iraq \\ Corresponding Author Email: Ali.Jassim@qu.edu.iq \\ Co-Author Email: Thaier.abid@qu.edu.iq
}

(Received 19/7/2017, Accepted 23/10/2017)

\begin{abstract}
The study was designed to compare the efficacy of using three injectable drugs; Ketamine, Paracetamol, and Tramadol in three anesthetic protocols. Fifteen apparently healthy adult sheep of either sex weighing (25-30 kg) were utilized, and divided into three equal groups; G1; $\left(K_{4} P_{10}\right)$ was given Ketamine $4 \mathrm{mg} / \mathrm{kg} \mathrm{B.W}+$ Paracetamol $10 \mathrm{mg} / \mathrm{kg} \mathrm{B.W,} \mathrm{G2;}\left(K_{4} T_{2}\right)$ was given Ketamine $4 m g / k g ~ B . W+$ Tramadol $2 m g / k g ~ B . W$, and $G 3 ;\left(K_{4} T_{2} P_{10}\right)$ was given Ketamine $4 \mathrm{mg} / \mathrm{kg} \mathrm{B.W}+$ Tramadol $2 \mathrm{mg} / \mathrm{kg}+$ Paracetamol $10 \mathrm{mg} / \mathrm{kg}$ B.W. Drugs were given to the animal by $I V$ injection for creating induction of anesthesia. The induction time, length of surgical anesthesia, recovery time, and degree of analgesia, degree of muscle relaxation, respiratory rate, heart rate and rectal temperature were recorded at 5 minutes before injection of drugs and consider as control readings. Readings were repeated at 5, 10, and 15 minutes after administration of anesthetic drugs. Results were displayed that anesthesia with $\left(K_{4} T_{2} P_{10}\right)$ protocol was better compared with the two other protocols as it produced reliable surgical anesthesia, good analgesia, muscle relaxation and short recovery time.
\end{abstract}

Keywords: Paracetamol, Tramadol, Ketamine, Anesthesia, Sheep.

\section{Introduction}

In general, ruminants successfully can be anesthetized by general anesthesia, with special efforts to avoid many difficulties that may accompanied ruminants anesthesia, like regurgitation, ruminal tympany, salivation, and cardiovascular and respiratory distress (1). Sheep is one species of ruminants frequently need to anesthetized with the surgical operations, and frequently used as a model of anesthesia of ruminants. The inhalation anesthesia, which is the best type of anesthesia, not always available in the field, and the use of the injectable anesthetics, is mandatory. Not all the anesthetic or the analgesic drugs are effective in all species of animals. The economic considerations and limited number of anesthetics and analgesics used in small ruminants may reflect arbiter the use of drug and technique (2) Ketamine is frequently used as an injectable general anesthetic drug in most species of animals, but it has unpleasant adverse effects related to the rigidity, and absence of muscle relaxation (3, $4,5)$. Paracetamol is the wide used analgesic in human beings, prescribed in a tablet forms. The combination between tramadol and Paracetamol give superior analgesic properties due to the synergistic analgesic effects of the two drugs because of the different site of action of each one lead to broad analgesic effect. Recently the introduce of the injectable type of Paracetamol (6) encourage researcher to share this drug as a part of anesthetic protocols. The study aim to evaluate the efficacy of use these three drugs in anesthesia of sheep. 


\section{Materials and Methods Ethical approval}

The Animal Ethical Committee of Veterinary Medicine College, University of AlQadisiyah, Iraq, has approved the present study under permission No: 427

The study was performed on 15 healthy adult local breed sheep of both sexes weighting $(27.4 \pm 2.46) \mathrm{kg}$. and their ages were range between (12-18 months). Animals were accommodated in the same environmental conditions, and were divided into three equal groups injected intravenously through the jugular vein with the anesthetic and analgesic drugs (drugs were mixed in the same syringe), while the animal in lateral recumbency. Animals were restrained by tying of the four limbs, and casting on lateral recumbency for 30 minutes before taking the reading of vital signs (The heart rate (HR), respiratory rate (RR), and rectal temperature (RT)), to reduce the disturbance of animal movement on the vital signs. The tie was released after giving the drugs. The experimental design include; $\mathrm{G} 1\left(\mathrm{~K}_{4} \mathrm{P}_{10}\right)$ was given Ketamine $4 \mathrm{mg} / \mathrm{kg}+$ Paracetamol $10 \mathrm{mg} / \mathrm{kg} \quad$ B.W, G2 $\left(\mathrm{K}_{4} \mathrm{~T}_{2}\right)$ was given Ketamine $4 \mathrm{mg} / \mathrm{kg}$ + Tramadol $2 \mathrm{mg} / \mathrm{kg} \mathrm{B.W}$, and $\mathrm{G} 3 \quad\left(\mathrm{~K}_{4} \mathrm{~T}_{2} \mathrm{P}_{10}\right)$ was given Ketamine $4 \mathrm{mg} / \mathrm{kg}+$ Tramadol $2 \mathrm{mg} / \mathrm{kg}+$ Paracetamol $10 \mathrm{mg} / \mathrm{k}$ B.W. (Paracetamol Injection 500 $\mathrm{mg} / 5 \mathrm{ml}$ Ajanta pharma. India, Tramadol-

\section{Results}

The HR reading was increased after 5 minutes from giving the anesthetic drugs more than the reading before anesthesia (time zero reading), in G1, G2 and G3 Figure (1), the reading of RR in all groups G1, G2, and G3 after 5 minutes of giving of drugs was significantly high and sharply increased than the control. In G2 the increase was more than double $(71.6 \pm 5.66)$ breath/minute, compared with (25.6 \pm 5.63$)$ breath / minute before anesthesia Figure (2).The rectal temperature in both G1 and G2 was decreased after 5 minutes of anesthesia than the control, while in G3 there was mild increase with no
Trabar-100 (Tramadol HCl), $100 \mathrm{mg} / 2 \mathrm{ml}$. mepha LLC Aesch-Basel, Switzerland, Ketamine: Ketamine $\mathrm{HCl}$ ampule: $500 \mathrm{mg} / 10$ ml. Astrapin, Germany). The vital parameters were taken for each animal, the normal respiratory rate (RR, breath/minutes) based on counting thoracic movement (7), heart rate (HR, beats/minute) by using stethoscope. Baseline recordings of RR, HR and RT, were recorded 5 minutes before drugs administration and considered \{(Time 0$)$ control reading of the same animal)\}, then at 5, 10, and 15 minutes after drug administration. The induction time, duration of anesthesia, duration of surgical anesthesia, degree of analgesia (by pinprick), degree of muscle relaxation was created by limb relaxation, by flexion and extension of the limbs, and graded to, no muscle relaxation = 0 (when the animal conscious), minimal muscle relaxation $=+1$, moderate muscle relaxation $=+2$, and marked muscle relaxation $=+3$ were reported (8), and recovery time were recorded for each animal.

\section{Statistical analysis:}

The obtained data were statistically analyzed using one way ANOVA test to find the significance between the times before and after drug administration and between the groups by using of SPSS program at the $\mathrm{P}<0.05$ (9).

significance Figure (3). The muscle relaxation was mild in G1, G2 and G3, while the limb analgesia and the flank analgesia were moderate in $\mathrm{G} 1$, more than mild in G2 and G3 Figure $(4,5,6)$. The rumen movement was remaining as such in G1, decreased in $\mathrm{G} 2$, and increased in $\mathrm{G} 3$ in compare with control (the reading before anesthesia) Figure(7). The induction time was 53.2 $\pm 3.2,60$, and $68 \pm 13.19$ seconds in G1, G2 and G3 respectively. The surgical anesthesia was $2.8 \pm 0.73,5$, and 5 minutes in G1, G2 and G3 respectively. The total time of recovery was $4.8 \pm 0.8,5.4 \pm 0.74$, and 


\section{Al-Qadisiyah Journal of Veterinary Medicine Sciences \\ (P-ISSN 1818-5746/ E-ISSN 2313-4429)}

www.qu.edu.iq/journalvm

$3 \pm 0.54$ minutes in G1, G2 and G3 respectively Figure (8).

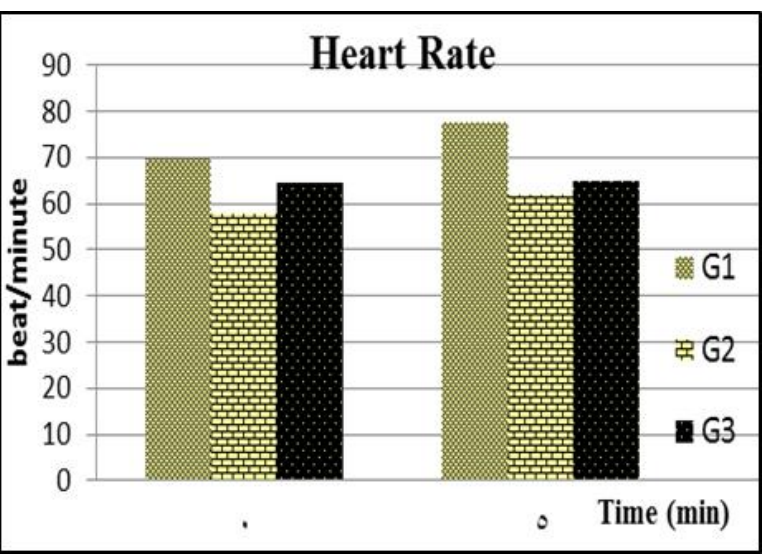

Figure (1): Effects of IV injected anesthetic protocols on heart rate (beat/minute): $\mathbf{G 1}\left(\mathrm{K}_{4} \mathrm{P}_{10}\right)$, G2 $\quad\left(K_{4} \mathbf{T}_{2}\right), \quad G 3\left(K_{4} \mathbf{T}_{2} P_{10}\right) . \quad K=K e t a m i n e$ $\mathbf{P}=$ Paracetamol, $\mathbf{T}=$ Tramadol

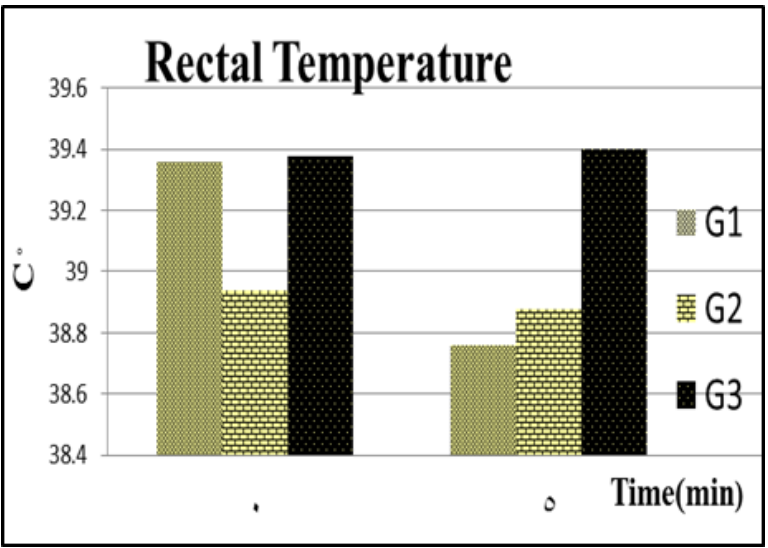

Figure. (3): Effects of IV injected anesthetic protocols on rectal temperature $(C)$ : $G 1\left(K_{4} P_{10}\right)$, G2 $\quad\left(K_{4} \mathbf{T}_{2}\right), \quad G 3 \quad\left(K_{4} \mathbf{T}_{2} \mathbf{P}_{10}\right) . \quad K=K e t a m i n e$ $\mathbf{P}=$ Paracetamol, $\mathbf{T}=$ Tramadol

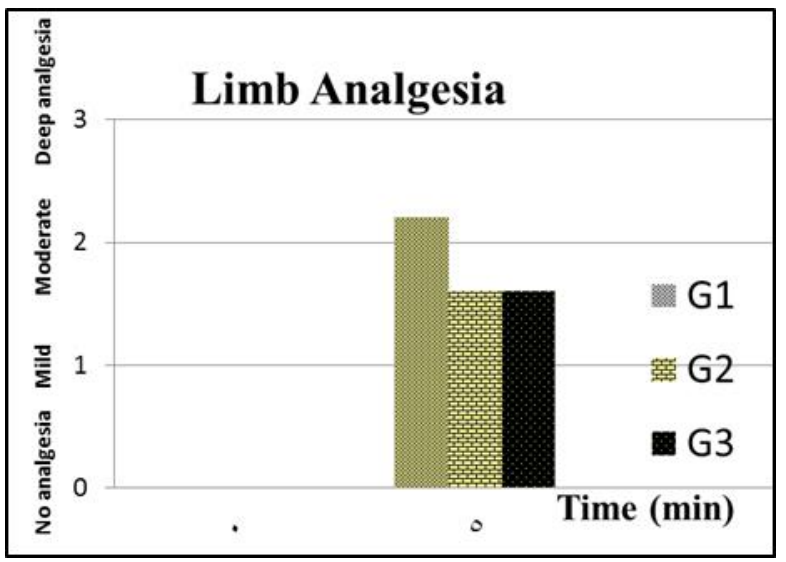

Figure (5): Effects of IV injected anesthetic protocols on limb analgesia: $G 1\left(K_{4} P_{10}\right), G 2\left(K_{4} T_{2}\right)$,

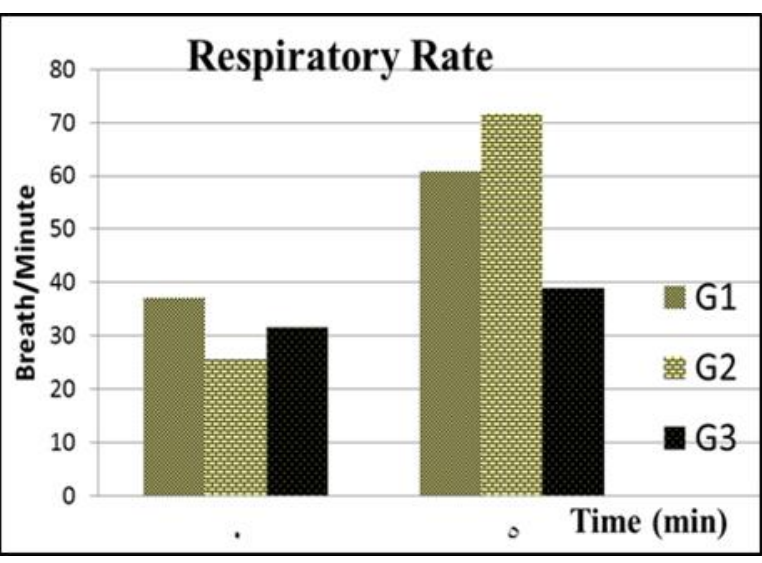

Figure (2): Effects of IV injected anesthetic protocols on respiratory rate (breath/minute): G1

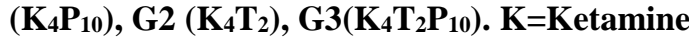
$\mathbf{P}=$ Paracetamol, $\mathbf{T}=$ Tramadol

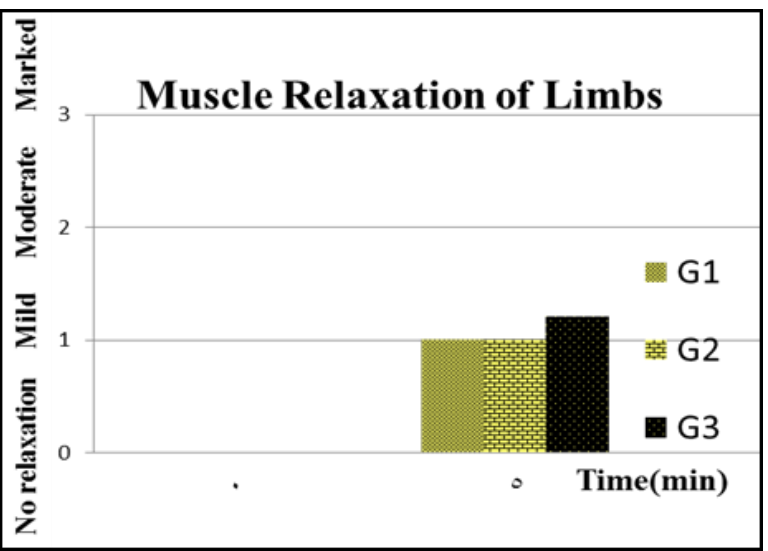

Figure. (4): Effects of IV injected anesthetic protocols on muscle relaxation: $\mathbf{G 1}\left(\mathrm{K}_{4} \mathbf{P}_{10}\right)$, G2 $\left(K_{4} T_{2}\right)$, $G 3\left(K_{4} T_{2} P_{10}\right)$. K=Ketamine $P=$ Paracetamol, $\mathrm{T}=$ Tramadol

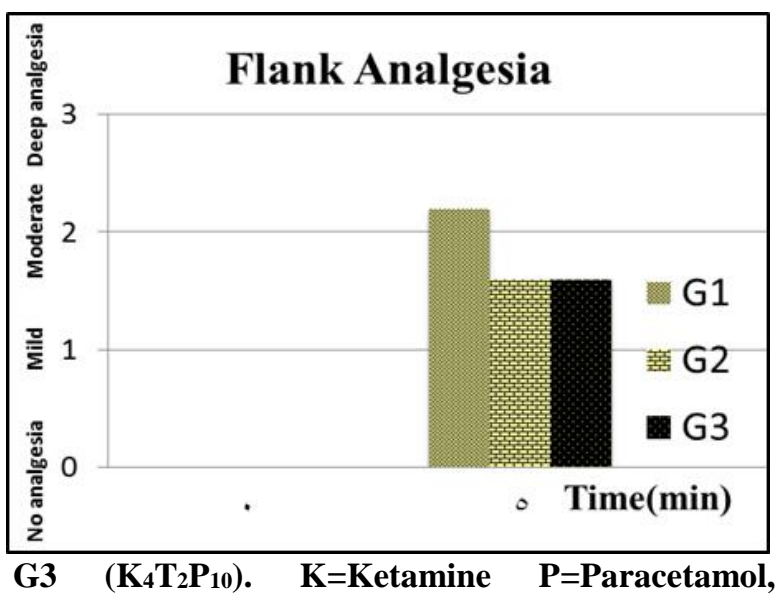

$\mathrm{T}=$ Tramadol 
Figure. (6): Effects of IV injected anesthetic protocols on flank analgesia: G1 $\left(\mathrm{K}_{4} \mathbf{P}_{10}\right)$, G2

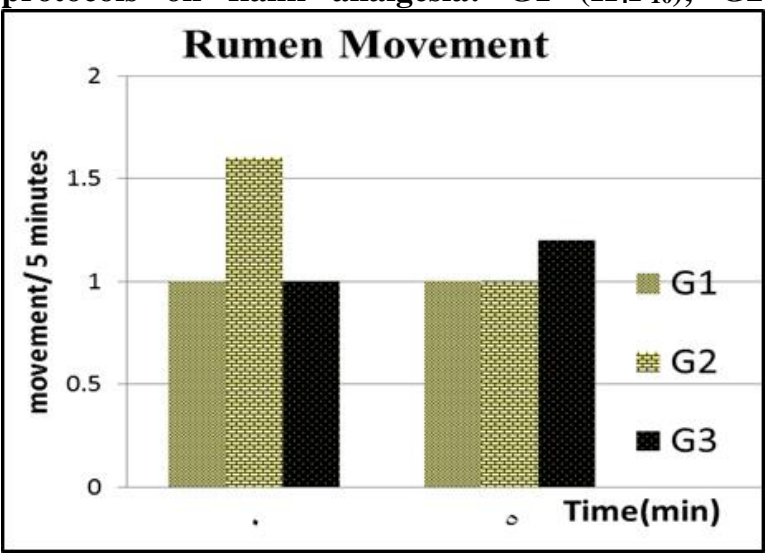

Figure (7): Effects of IV injected anesthetic protocols on rumen movement: G1 $\left(\mathrm{K}_{4} \mathbf{P}_{10}\right)$, G2 $\left(K_{4} T_{2}\right)$, G3 $\left(K_{4} T_{2} P_{10}\right)$. $K=$ Ketamine $P=$ Paracetamol, T=Tramadol

\section{Discussion}

Restricted anesthetic and analgesic drugs approved to use in sheep, and the costeffective may orient the use of the drug and the technique. In the field, unless the cost of the animal is high, the use of inhalational anesthesia is difficult and costly. Injectable anesthesia is easy and relatively safe to perform. Injectable anesthetic drugs are used for either induction or for maintenance of short-term anesthesia. Induction of anesthesia with IV anesthetic drugs is preferable for inhalational anesthetics in ruminants because it reduces the incidence of regurgitation and aspiration of ruminal contents (2). The induction, duration, and depth of sedation, analgesia, and anesthesia are depend on the route and rate of administration of these injectable drugs. The IV route is the fastest for giving the effect, but is the shortest in duration (3). Introduce of multiple drugs in anesthesia to minimize the dose and therefore the side effects of each one is called balanced anesthesia (10). It has been widely used for many years, and nowadays this term moved to pain medication, thus called balanced analgesia $(11,12)$. Using fewer doses from different classes of drugs, each drug has special target pain mechanism gives effective analgesia and reduce the related dose side
$\left(K_{4} T_{2}\right), G 3\left(K_{4} T_{2} P_{10}\right)$. K=Ketamine $P=$ Paracetamol, T=Tramadol

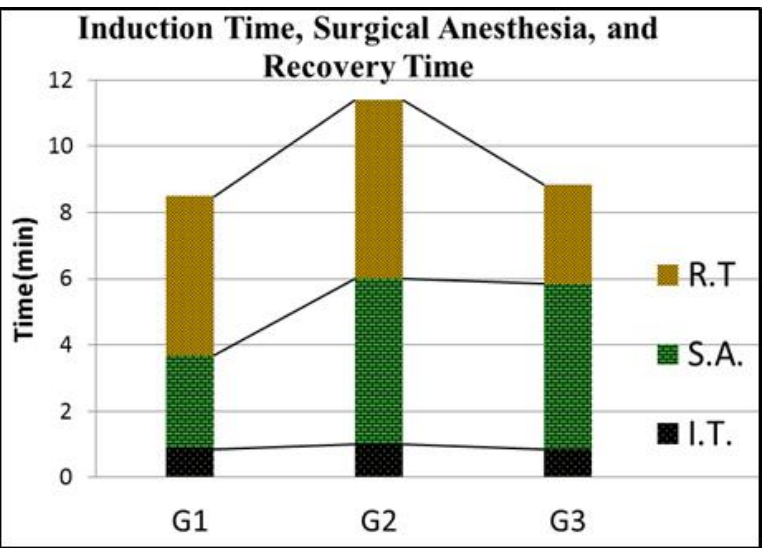

Figure (8): Effects of IV injected anesthetic protocols on Induction time (IT), Surgical anesthesia (SA), and Recovery time (RT): G1 $\left(K_{4} P_{10}\right)$, G2 $\left(K_{4} T_{2}\right), G 3\left(K_{4} T_{2} P_{10}\right)$. K=Ketamine $\mathbf{P}=$ Paracetamol, $\mathbf{T}=$ Tramadol

effect. Merges between these drugs breadth the area of analgesia. This method propose increased efficiency of these drugs due to the additive or synergistic effects without increasing the dose. Model of combination procedure would both enhance analgesic effectiveness and reduce the side effects in compare with using each drug alone (13). In this experiment, the total duration of anesthesia is 5 minutes in the $(\mathrm{G} 1, \mathrm{G} 2$, and G3) where the anesthetic drugs given IV. The recorded vital signs (HR, RR, RT) are assigned in 5 minutes only due to the short duration of anesthesia of these protocols in these doses by the IV route of administration, that means these doses by the IV route is suitable only for induction of anesthesia. This time is sufficient to do endotracheal intubation if the maintenance of anesthesia is maintained by inhalation anesthesia, or maintained by total IV anesthesia. The induction time in these three protocols is $53.2,60$, and 68 seconds. It is fast and good induction time; bring about a crossing the dangerous side effects may occur in the early stages of anesthesia. There is little calculating difference in induction time between G1, and G2, where the paracetamol or tramadol is added to the ketamine. The 
induction time in G3 is significantly difference than what in $\mathrm{G} 1$, there is no explanation for this and it may need more investigation in future. The surgical anesthesia in G1 (2.8 minutes) which is significantly shorter than G2, and G3. It seem that the paracetamol not increase the anesthetic effect of ketamine. It is not like the tramadol in G2 and G3. This result is in agreement with (14) who found the tramadol improve the quality of ketamine anesthesia in pigs. Also it is compatible with (15), who find the combination between tramadol and ketamine produce significant synergistic interactions with less CNS depression compare with equine analgesic dose of ketamine. The HR and RR are increased after 5 minutes of giving the three anesthetic protocols. Ketamine act as a respiratory stimulant, or at least not like most general anesthetic drugs, which cause respiratory depression in spontaneous breathing patients, also the ketamine act as bronchodilator and increase the lung compliance and decrease airway resistance. Ketamine appears to stimulate the cardiovascular system, producing increase in the heart rate, cardiac output and the blood pressure, due to the sympathomimetic effects of ketamine in blocking the reuptake of catecholamine (16, 17). Paracetamol is an effective and welltolerated analgesic with an excellent safety profile within the therapeutic dose range up to $4 \mathrm{~g}$ /day in human beings. No adverse

\section{References}

1-Valverde A, Doherty TJ. Anesthesia and analgesia in ruminants. In: Fish R E, Brown M J, Danneman PJ, Karas AZ (eds.) Anesthesia and Analgesia in Laboratory Animals ( $\left.2^{\text {nd }} e d.\right)$ (2008) Elsevier Inc. pp 385-411.

2-Galatos AD. Anesthesia and analgesia in sheep and goats. Vet. Clin. Food Anim. (2011) 27: 47-59

3-Clarke KW, Trim CM, Hall LW. Anesthesia of sheep, goats and other herbivores. In: Veterinary Anesthesia. $11^{\text {th }}$ edition (2014). Elsevier Ltd. Pp. 346-367

4-Greene SA. Protocols for anesthesia of cattle. Vet. Clin. North Am. Food Anim. Pract. (2003) 19, 679-693. effects are seen on the cardiovascular and pulmonary system at the therapeutic doses in human beings, the only side effect seen is the liver damage or failure occur after over dosage (18). In this study there is marked increase in RR in paracetamol ketamine group, and due to less studies on paracetamol in animals, we do not find explanations for this result. It may have direct stimulant effect on the respiratory center in sheep. This point needs more explanations in future. Tramadol in man and some species of animal does not have adverse effect on cardiovascular or respiratory systems $(19,20)$. In dogs, tramadol does not cause depression of heart or respiratory rates following intravenous administration in dosage ranges between (1, 2 , and $4 \mathrm{mg} / \mathrm{kg}$ ), and the femoral pulses remained strong throughout the assessment period (21). In this study, the respiratory rate is increased more than doubling after 5 minutes of giving tramadol in G2. There seem to be a range of activity in different species, which may be associated with active metabolite formation (22). The rectal temperature in G1 and G2 is decreased, and in G3 there is non- significant mild increase after 5 minutes of giving of anesthetic drugs. This result did not agree with the previous studies, and no explanations for this result. The muscle relaxation is mild, and the analgesia is nearly moderate. This may be attributed to the low doses of the drugs in these three anesthetic protocols.

5-Seddighi R, Doherty $\mathrm{T}$ J. Field sedation and anesthesia of ruminants. Veterinary Clinics of North America: Food Animal Practice (2016), 32 (3): $553-570$

6-Duggan ST, Scott LJ. Intravenous paracetamol (Acetaminophen). Drug. (2009) 69 (1):101-113.

7-Afshar FS, Baniadam A, Marashipour SP. Effect of xylazine ketamine on arterial blood pressure, arterial blood $\mathrm{pH}$, blood gas, rectal temperature, heart and respiratory rates in goats. Bull. Vet. Inst. Pulawy. (2005) 49:481-484.

8-Lin HC, Wallace SS, Tyler JW, Robbins RL, Thurmon JC, Wolfe DF. Comparison of tiletamine-zolazepam-ketamine and tiletamine- 
zolazepam-ketamine-xylazine anesthesia in sheep. Aust. Vet. J. (1994) 71(8):239-242.

9-SPSS. Statistical package for social science version 16 (win/ Mac/ Linux) user's guide Inc-Chicago ш, USA. (2008) Website http: // www.spss.com.

10-Muir WW, Yamashita K. Balanced anesthesia in horses. AAEP Proceedings (2000) 46: 98-99

11-Kehlet H, Dahl JB. The value of (Multimodal) or (Balanced Analgesia) in postoperative pain treatment. Anesth. Analg. (1993) 77:1048-1056

12-Schug SA. Combination analgesia in 2005 a rational approach: focus on paracetamol-tramadol. Clin. Rheumatol. (2006) 25 (Suppl. 1): S16-S21

13-Valverde A. Balanced anesthesia and constant-rate infusions in horses. Veterinary Clinics of North America: Equine Practice. (2013) 29 (1): 89-122

14-Ajadi RA, Olusa TA, Smith OF, Ajibola ES, Adeleye OE, Adenubi OT, Makinde FA. Tramadol improved the efficacy of ketamine-xylazine anesthesia in young pigs. Vet. Anesth. Analg. (2009) 36:562-566.

15-Chen Y, Chan SY, Ho PC. Isobolographic analysis of the analgesic interactions between ketamine and tramadol. Journal of Pharmacy and Pharmacology (2002) 54 (5): 623-631

16-Haas DA, Harper DG. Ketamine: A review of its pharmacologic properties and use in ambulatory anesthesia. Anesth. Prog. (1992) 39: 61-68.

17-Bergman SA. Ketamine: Review of its pharmacology and its use in pediatric anesthesia. Anesth. Prog. (1999) 46: 10-20.

18-Raffa R. Pharmacological aspects of successful long-term analgesia. Clin. Rheumatol. (2006) 25 (Suppl. 1): S9-S15

19-Scott LJ, Perry CM. Tramadol: a review of its use in perioperative pain. Drugs. (2000) 60 (1):139176.

20-Livingston A. Pain and analgesia in domestic animals. Handbook of Experimental Pharmacology. (2010) 199: 159-190

21-McMillan C, Livingston A, Clarke CR, Dowling PM, Taylor SM, Duke T, Terlinden R. Pharmacokinetics of intravenous tramadol in dogs. Can. J. Vet. Res. (2008) 72:325-331

22-Pypendop BH, Ilkew JE. Pharmacokinetics of tramadol and its metabolite O-desmethyl-tramadol in cats. J. Vet. Pharmacol. Ther. (2008) 31:52-59 